\title{
Sudden Iatrogenic Blindness in Patients Treated with Biological Cancer Therapy
}

\section{Maaly Abdel Halim Abdel Fattah",2,3*, Fatima Babiker Ali Babiker ${ }^{3}$ and Selwa Abdulla Alhazzaa ${ }^{2,3}$}

${ }^{1}$ Ophthalmology Department, Cairo University, College of medicine, Cairo, Egypt

${ }^{2}$ Ophthalmology Department, King Faisal Specialist Hospital and Research Center, Riyadh, Saudi Arabia

${ }^{3}$ Al-Faisal University college of Medicine, Riyadh, Saudi Arabia

*Corresponding Author: Maaly Abdel Halim Abdel Fattah, Ophthalmology

Department, Cairo University, College of medicine, Cairo, Egypt and

Ophthalmology Department, King Faisal Specialist Hospital and Research

Center and Al-Faisal University college of Medicine, Riyadh, Saudi Arabia.

DOI: 10.31080/ASOP.2020.03.0096
Received: February 02, 2020

Published: February 24, 2020

(C) All rights are reserved by Maaly Abdel

\section{Halim Abdel Fattah., et al.}

\begin{abstract}
Sunitinib and Ponatinib are two biological drugs that have greatly contributed to the treatment advances of different types of cancer. However, the ocular adverse effects of those drugs were not well studied and only a few case reports, within the literature, described patients who developed vision loss and ocular changes.

In this study, we reported the clinical presentation of the first two Saudi patients presenting with a sudden loss of vision due to recent lines of treatment. The effect of these drugs has strongly implied the importance of cautious use and the need for further studies on their ocular side effects.
\end{abstract}

Keywords: Blindness; Ocular; Biological; Sunitinib; Ponatinib and Anticancer

\section{Abbreviations}

CML: Chronic Myeloid Leukaemia; TKI: Tyrosine Kinase Inhibitors; FDA: Food and Drug Administrationl; CT: Computed Tomography; OD: Oculus Dexter; OS: Oculus Sinister; OU: Oculus Uterque; OCT: Optical Coherence omography; CNS: Central Nervous System And CSF: Cerebral Spinal Fluid

\section{Introduction}

Biological cancer drugs have been evolving rapidly over the past decade and became one of the great contributors to the advancement of cancer therapy. Ponatinib was approved for the treatment of adult patients with any phase of CML or Philadelphia chromosome-positive acute lymphoblastic leukaemia that is resistant or intolerant to prior TKI therapy. Additionally, Sunitinib was approved by FDA in 2006 as the first-line therapy for advanced renal cell carcinoma and gastrointestinal stromal tumour. It is a nov- el oral multi-targeted tyrosine kinase inhibitor with anti-tumour and anti-angiogenic activities [1].

The current question now regarding these new medications is whether patients can tolerate their side effects. Especially if it involves major functions that significantly reduces the quality of a patient's life, such as their own vision.

\section{Case Description}

Case one

54-year-old hypertensive female patient diagnosed as having metastatic clear cell carcinoma post-discovery of a renal mass in 2015. There is no family history of malignancy or Blood disorders. Accordingly, the patient was started on multiple cycles of Sunitinib and Bevacizumab. Each cycle composed of 50mg daily Sunitinib for 4 weeks followed by two weeks off drug. Bevacizumab was given 
on the 28th and 29th day of each cycle with a $10 \mathrm{mg} / \mathrm{kg}$ dose (except the first cycle which included a $5 \mathrm{mg} / \mathrm{kg}$ dose).

In the following months, follow up CT showed stable disease and her laboratory markers (renal, hepatic and coagulation profiles) where all within normal limits. However, the patient complained of decreased vision in her right eye which dropped to 20/400. Dilated fundus examination showed a single spot of epi-macular haemorrhage with a normal ocular exam of the left eye (Figure 1).

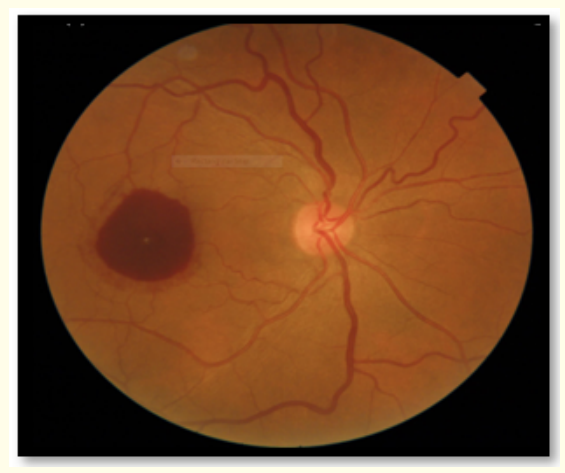

Figure 1: Fundus photo of the right eye of patient that used Sunitinib.

The patient's vision on the right eye improved significantly after 2 months of discontinuation of Sunitinib with complete absorption of retinal haemorrhage.

\section{Case two}

28-year-old male patient having B cell lymphoblastic lymphoma in 2016 based on cytogenic analysis, karyotyping and FISH testing which were positive for BCR-ABL translocation. Thus, the patient was given Dasatinib, weekly Vincristine and intrathecal Methotrexate with Dexamethasone. Upon remission, the patient underwent a matched-sibling donor bone marrow transplant from his sister for which he tolerated well with no complications. Unfortunately, a year later, the patient developed recurrence and was given Vincristine and Ponatinib and his immunosuppressant drugs were stopped.

In late 2018, the patient developed sudden loss of vision (no perception of light) in the left eye. Visual assessment revealed a left central retinal artery and vein occlusion. Therefore, Ponatinib was discontinued as it is known to cause arterial/venous thrombosis. Two days later the patient presented to the Emergency Department complaining of a sudden loss of vision in the other eye. Ocular assessment revealed fixed dilated pupils OU with hyperemic optic disk with irregular elevated margins, cherry red spot with congested lower temporal retinal vessels and attenuated segmented upper temporal and nasal retinal arteries and veins. (Figure 2) A coagulation profile was done for the patient to exclude other bleeding disorders. However, the results did not reveal any underlying coagulopathy. Despite the discontinuation of Ponatinib, the patient's vision did not improve and the fundoscopic exam showed permanent structural damage.

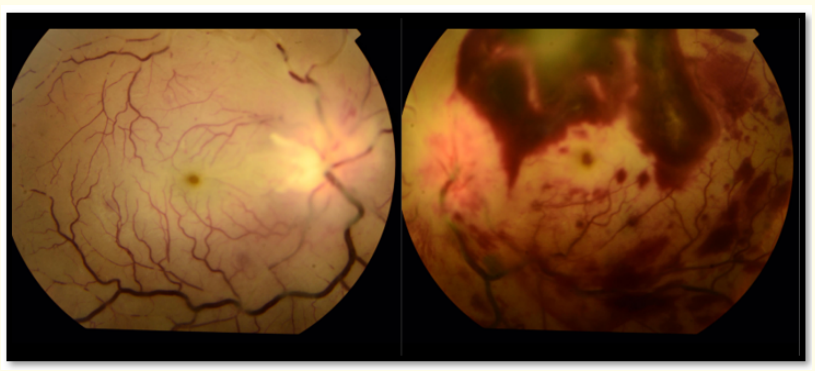

Figure 2: Fundus photo of patient that used Ponatinib.

\section{Discussion}

In this study, we described the medical course of the first two Saudi patients treated with Ponatinib and Sunitinib who developed visual insults as a complication of the treatment.

This conclusion was extracted after additional investigations revealing no other possible vascular causes for their ocular adverse events.

To our knowledge, this is the first study to report those ocular side effects of Sunitinib and Ponatinib in Saudi Arabia.

However, there have been cases reported in other countries such as Germany, Canada and the United States.

In Germany: Wegner and Khoramnia reported a 52-year-old male diagnosed with metastatic renal cell carcinoma who presented with decreased visual acuity in both eyes in 2018. The patient was on Sunitinib $50 \mathrm{mg}$ daily for 3 weeks. His examination 
revealed visual acuity of $6 / 12$ in both eyes with OCT revealed bilateral neurosensory retinal detachment bilaterally. Sunitinib was stopped and the patient appeared two weeks later with improved visual acuity of $6 / 6$ bilaterally. His OCT revealed a complete resolution of the neurosensory retinal detachment [2].

Another study done by Fraunfelder., et al. who reported 24 cases of retinal detachment secondary to Sunitinib administration [3].

As for Ponatinib, newly identified complications of vision loss and ocular vascular thrombosis appeared in the phase two trials during 48 months of follow-up of patients who were treated with Ponatinib. According to the US FDA, 6\% of Ponatinib-treated patients developed blurry vision and $2 \%$ developed retinal vein occlusion, macular oedema and retinal haemorrhage [1].

In addition, Elizondo Leal., et al. reported in Canada a 63-yearold male known case of chronic myeloid leukaemia with CNS involvement. The patient was initially treated with Cytarabine intrathecally for which he responded well. Yet he later relapsed, necessitating the switch to Blinatumomab plus Ponatinib. During the treatment the patient developed many adverse effects to Ponatinib including peripheral neuropathy, worsening of blood pressure (systolic pressure above 200) and blurry vision. Upon examination the patient appeared to have decreased vision in the left eye and laboratory, CSF and imaging investigation showed no signs of recurrent CML. Thus, a diagnosis of Ponatinib induced ocular toxicity was made [4].

\section{Conclusion}

The Ocular adverse effects of the recently approved tyrosine kinase inhibitors (Sunitinib and Ponatinib) can be severely detrimental to the vision of treated patients and reduce the patient's quality of life. Further studies are needed to explore the potential ocular side effects of those medications and possible approaches to prevention. Moreover, Physicians need to practice caution during the administration of those biological treatment and weigh the benefits and risks for each patient individually.

\section{Ethics Approval and consent to participate}

The two patients signed consent to participate in our study.

\section{Consent for Publication}

Research centre of King Faisal Specialist centre approved our study and agree for publication.

\section{Availability of data and Material}

All data generated or analysed during the study are included in the published article.

\section{Competing Interests}

The author has no commercial interest in any materials discussed in this article.

\section{Funding}

None.

\section{Bibliography}

1. Centre for Drug Evaluation and Research. "FDA Requires Multiple New Safety Measures for Leukaemia Drug (www.Iclusig. com)" U.S. Food and Drug Administration (2018).

2. Wegner A and R Khoramnia. "Neurosensory Retinal Detachment Due to Sunitinib Treatment". Eye 25.11 (2011): 15171518.

3. Fraunfelder Frederick T., et al. "Oral Anti-Vascular Endothelial Growth Factor Drugs and Ocular Adverse Events." Mary Ann Liebert, Inc., Publishers 34.6 (2018): 632-635.

4. Elizondo Leal José A., et al. "Optic Neuropathy in Extramedullary, Blast Crisis of Chronic Myeloid Leukemia". Canadian Journal of Ophthalmology. Journal Canadien D'ophtalmologie, U.S. National Library of Medicine (2019).

\section{Assets from publication with us}

- Prompt Acknowledgement after receiving the article

- Thorough Double blinded peer review

- Rapid Publication

- Issue of Publication Certificate

- High visibility of your Published work

Website: $\underline{w w w}$.actascientific.com/

Submit Article: www.actascientific.com/submission.php

Email us: editor@actascientific.com

Contact us: +919182824667 